\title{
Notation And Special Symbols
}

$\oplus$ Gyroaddition, Gyrogroup operation.

$\ominus$ Gyrosubtraction, Inverse gyrogroup operation.

$\boxplus$ Cogyroaddition, Gyrogroup cooperation.

$\boxminus$ Cogyrosubtraction, Inverse gyrogroup cooperation.

$\oplus_{\mathrm{E}}$ Einstein addition (of relativistically admissible coordinate velocities, and generalizations).

$\ominus_{\mathrm{E}}$ Einstein subtraction.

$\boxplus_{\mathrm{E}}$ Einstein coaddition.

$\boxminus_{\mathrm{E}}$ Einstein cosubtraction.

$\oplus_{\mathrm{M}}$ Möbius addition.

$\ominus_{\mathrm{M}}$ Möbius subtraction.

$\boxplus_{\mathrm{M}}$ Möbius coaddition.

$\boxminus_{\mathrm{M}}$ Möbius cosubtraction.

$\oplus_{\mathrm{U}}$ PV addition (of relativistically admissible proper velocities, and generalizations).

$\ominus_{\mathrm{U}}$ PV subtraction.

$\boxplus_{\mathrm{U}}$ PV coaddition.

$\boxminus_{\mathrm{U}}$ PV cosubtraction.

$\boxplus_{k}$ Einstein coaddition of $k$ th order, (10.72).

$\otimes$ Scalar multiplication (scalar gyromultiplication) in a gyrovector space.

$\otimes_{\mathrm{E}}$ Einstein scalar multiplication.

$\otimes_{\mathrm{M}}$ Möbius scalar multiplication.

$\otimes_{\mathrm{U}}$ PV scalar multiplication.

$\diamond$ Gyropolygonal gyroaddition, Definition 2.17.

$:=$ Equality, where the lhs is defined by the rhs.

$=$ : Equality, where the rhs is defined by the lhs. 


\section{$(\ldots)^{t}$ Transpose of $(\ldots)$.}

$\mathbf{a b}$ A segment with distinct endpoints $\mathbf{a}$ and $\mathbf{b}$ of (i) a gyroline (gyrosegment), or (ii) a cogyroline (cogyrosegment). A gyroline (cogyroline) containing the distinct points $\mathbf{a}$ and $\mathbf{b}$.

$|\mathbf{a b}|$ Length of (i) a gyrosegment (gyrolength), or (ii) a cogyrosegment (cogyrolength).

abc A gyrotriangle with vertices $\mathbf{a}, \mathbf{b}$ and $\mathbf{c}$.

abdc A gyroquadrilateral, particularly a gyroparallelogram, with vertices $\mathbf{a}, \mathbf{b}, \mathbf{d}$ and $\mathbf{c}$.

$\mathbf{m}_{\text {abdc }}$ Gyroparallelogram Gyrocenter.

$\mathbf{a b c d a}^{\prime} \mathbf{b}^{\prime} \mathbf{c}^{\prime} \mathbf{d}^{\prime}$ Gyrparallelepiped, Fig. 10.13, or parallelepiped, Fig. 10.11, with its 8 indicated vertices.

a $a=\|\mathbf{a}\|$ is the gyrolength of gyrovector $\mathbf{a}$.

$a_{s} \quad a_{s}=a / s$, etc., in a gyrovector space $\left(\mathbb{V}_{s}, \oplus, \otimes\right)$.

$\mathbf{a}_{s} \mathbf{a}_{s}=\mathbf{a} / s$, etc., in a gyrovector space $\left(\mathbb{V}_{s}, \oplus, \otimes\right)$.

$\mathbf{a}_{\gamma}$ The gamma correction of a, etc. Eqs. (8.93), p. 291: (8.95).

$\mathbf{a}_{\beta}$ The beta correction of a, etc. Eqs. (8.93), p. 291; (8.95).

Aut An automorphism group.

Aut $t_{0}$ A subgroup of an automorphism group.

CM Center of Momentum, and Center of Mass, collectively.

CMM Center of MomentuM.

CMS Center of MasS.

$c$ The vacuum speed of light.

$d_{\oplus} d_{\oplus}(\mathbf{a}, \mathbf{b})=\|\mathbf{b} \ominus \mathbf{a}\|$.

$d_{\boxplus} d_{\boxplus}(\mathbf{a}, \mathbf{b})=\|\mathbf{b} \boxminus \mathbf{a}\|$.

gyr Gyrator. gyr $[\mathbf{a}, \mathbf{b}]$ the gyration (gyroautomorphism) generated by $\mathbf{a}$ and $\mathbf{b}$.

ILT Inhomogeneous Lorentz Transformation.

$s$ Gyrovector space analogue of the vacuum speed of light $c$. It is the radius of the ball in ball models of hyperbolic geometry and their gyrogroups and gyrovector spaces.

$\mathbf{u} \times \mathbf{v}$ Vector product of $\mathbf{u}, \mathbf{v} \in \mathbb{R}^{3}$ with components $(\mathbf{u} \times \mathbf{v})_{1}$ etc.

$\gamma_{\mathbf{v}}$ The gamma factor, $\gamma_{\mathbf{v}}=\left(1-\|\mathbf{v}\|^{2} / s^{2}\right)^{-1 / 2}$ in the ball $\mathbb{V}_{s}$.

$\beta_{\mathbf{v}}$ The beta factor, $\beta_{\mathbf{v}}=\left(1+\|\mathbf{v}\|^{2} / s^{2}\right)^{-1 / 2}$ in the ball $\mathbb{V}_{s}$.

$\mathbb{B}^{3} \mathbb{B}^{3}=\mathbb{R}_{s=1}^{3}$ is the open unit ball of the Euclidean 3-space $\mathbb{R}^{3}$.

$\mathbb{B}^{n} \mathbb{B}^{n}=\mathbb{R}_{s=1}^{n}$ is the open unit ball of the Euclidean n-space $\mathbb{R}^{n}$.

$\partial \mathbb{B}^{n}$ The boundary of the ball $\mathbb{B}^{n}$.

$I$ Identity automorphism.

$i \sqrt{-1}$ 
$\mathbb{N}$ The set of all natural numbers $\{1,2,3, \ldots\}$.

$\mathbb{R}$ The real line.

$\mathbb{R}^{>0}$ The positive ray of the real line $\mathbb{R}$.

$\mathbb{R}^{\geq 0}$ The nonnegative ray of the real line $\mathbb{R}$.

$\mathbb{R}^{n}$ The Euclidean $n$-space.

$\mathbb{R}_{s}^{n}$ The $s$-ball of the Euclidean $n$-space, $\mathbb{R}_{s}^{n}=\left\{\mathbf{x} \in \mathbb{R}^{n}:\|\mathbf{x}\|<s\right\}$. $S O(n)$ The group of all rotations of $\mathbb{R}^{n}$ about its origin.

$\mathbb{S}_{s}$ The cone $\mathbb{S}_{s}=\left\{(t, \mathbf{x})^{t}: t \in \mathbb{R}^{>0}, \mathbf{x} \in \mathbb{V}\right.$, and $\left.\mathbf{v}=\mathbf{x} / t \in \mathbb{V}_{s}\right\}$ $(S,+)$ A groupoid, a set $S$ with a binary operation + .

$\mathbb{V}$ A real inner product space $\mathbb{V}=(\mathbb{V},+, \cdot)$ with a binary operation + and an inner product $\cdot$

$\mathbb{V}_{s}$ The $s$-ball of the real inner product space $\mathbb{V}$.

$\mathbf{v}$ In most cases $\mathbf{v}$ is a generic element of $\mathbb{V}_{s}$. Similarly are $\mathbf{a}, \mathbf{b}, \mathbf{c}, \ldots$ and $\mathbf{u}, \mathbf{w}$ and $\mathbf{x}, \mathbf{y}, \mathbf{z}$.

$\mathbf{v}_{s} \quad \mathbf{v}_{s}=\mathbf{v} / s,\|\mathbf{v}\|_{s}=\|\mathbf{v}\| / s$, etc.

$\mathbb{Z}$ The set of all integers $\{\ldots-3,-2,-1,0,1,2,3, \ldots\}$. 
This page intentionally left blank 


\section{Bibliography}

Carl G. Adler. Does mass really depend on velocity, dad? Amer. J. Phys., 55(8):739-743, 1987.

Lars V. Ahlfors. Conformal invariants: topics in geometric function theory. McGraw-Hill Book Co., New York, 1973. McGraw-Hill Series in Higher Mathematics.

Lars V. Ahlfors. Möbius transformations in several dimensions. University of Minnesota School of Mathematics, Minneapolis, Minn., 1981.

Lars V. Ahlfors. Old and new in Möbius groups. Ann. Acad. Sci. Fenn. Ser. A I Math., 9:93-105, 1984.

David Alba, Luca Lusanna, and Massimo Pauri. Centers of mass and rotational kinematics for the relativistic $N$-body problem in the rest-frame instant form. J. Math. Phys., 43(4):1677-1727, 2002.

Joseph B. Altepeter, Daniel F. V. James, and Paul G. Kwiat. Qubit quantum state tomography. In Quantum state estimation, volume 649 of Lecture Notes in Phys., pages 113-145. Springer, Berlin, 2004.

Simon L. Altmann. Rotations, quaternions, and double groups. The Clarendon Press Oxford University Press, New York, 1986.

J. Anandan and L. Stodolsky. On the relation between quantum mechanical and classical parallel transport. Phys. Lett. A, 266(2-3):95-97, 2000.

James L. Anderson. Principles of relativity physics. Academic Press, New York, 1967.

George Arfken. Mathematical methods for physicists. Academic Press, New York, 1985

Arvind, K. S. Mallesh, and N. Mukunda. A generalized Pancharatnam geometric phase formula for three-level quantum systems. J. Phys. A, 30(7):24172431, 1997.

Neil Ashby. Relativity in the global positioning system. Living Rev. Relativity, 6(1), 2003. Online articel, [http://relativity.livingreviews.org/Articles/lrr2003-1].

John Bahcall, Tsvi Piran, and Steven Weinberg (eds.). Dark Matter in the Universe. sec. ed. Kluwer Academic Publishers Group, Dordrecht, 2004.

J. F. Barrett. Special relativity and hyperbolic geometry. Univ. Sunderland, Sun- 
derland, UK, 1998. Physical Interpretations of Relativity Theory. Proceedings, London, UK, 11-14 Sept. 1998.

J. F. Barrett. On Carathéodory's approach to relativity and its relation to hyperbolic geometry. In Constantin Carathéodory in his ... origins (VissaOrestiada, 2000), pages 81-90. Hadronic Press, Palm Harbor, FL, 2001.

Claus Beisbart and Tobias Jung. The messy mass? On the concept of mass in special relativity. Philos. Natur., 41(1):1-52, 2004.

Jacob D. Bekenstein. The modified Newtonian dynamics - MOND and its implications for new physics. Contemp. Physics, 47(6):387-403, 2007.

Michel Le Bellac. Quantum information and quantum computation. Cambridge University Press, Cambridge, 2006.

Lanfranco Belloni and Cesare Reina. Sommerfeld's way to the thomas precession. European J. Phys., 7:55-61, 1986.

Ingemar Bengtsson and Karol Życzkowski. Geometry of quantum states: An introduction to quantum entanglement. Cambridge University Press, Cambridge, 2006.

Marcel Berger. Geometry. I. Universitext. Springer-Verlag, Berlin, 1987. Translated from the French by M. Cole and S. Levy.

F. J. Bloore. Geometrical description of the convex sets of states for systems with spin- 1/2 and spin-1. J. Phys. A, 9(12):2059-2067, 1976.

Karl Blum. Density matrix theory and applications. Plenum Press, New York, second edition, 1996.

Émile Borel. La cinématique dans la théorie de la relativité. Comptes Rendus des séances de l'Academie des Sceinces, 157:703-705, 1913.

Emile Borel. Introduction Géométrique a Quelques Théories Physiques. GauthierVillars, Paris, 1914.

Alexey V. Borisov and Ivan S. Mamaev. The restricted two-body problem in constant curvature spaces. Celestial Mech. Dynam. Astronom., 96(1):1-17, 2006.

M. Born and K. Fuchs. The mass centre in relativity. Nature, 145:587, 1940.

Andrzej Borowiec, Włodzimierz Godłowski, and Marek Szydłowski. Dark matter and dark energy as effects of modified gravity. Int. J. Geom. Methods Mod. Phys., 4(1):183-196, 2007.

O. Bottema. On the medians of a triangle in hyperbolic geometry. Canad. J. Math., 10:502-506, 1958.

Carl B. Boyer. History of analytic geometry. Dover Publications Inc., Mineola, NY, 2004. Reprint of the 1956 original.

James Bradlay. A letter from the Reverend Mr. James Bradly to Dr. Edmund Halley giving an account of a new discovered motion of the fixed stars. Philos. Trans. R. Soc. London, 35: 637-661, 1728.

Ph. Brax. The cosmological constant problem. Contemp. Physics, 45(3):227-236, 2004.

James W. Cannon, William J. Floyd, Richard Kenyon, and Walter R. Parry. Hyperbolic geometry. In Flavors of geometry, pages 59-115. Cambridge Univ. Press, Cambridge, 1997.

C. Carathéodory. Conformal Representation. Dover, 1998. Unabridged republi- 
cation of the 1932 work.

C. Carathéodory. Theory of functions of a complex variable. Vol. 2. Chelsea Publishing Company, New York, 1954. Translated by F. Steinhardt.

Michael A. Carchidi. Generating exotic-looking vector spaces. College Math. J., 29(4):304-308, 1998.

Manfredo Perdigao do. Carmo. Differential geometry of curves and surfaces. Prentice-Hall, 1976.

Jing-Ling Chen and Mo-Lin Ge. On the Wigner angle and its relation with the defect of a triangle in hyperbolic geometry. J. Geom. Phys., 25(3-4):341345, 1998.

Jing-Ling Chen and Abraham A. Ungar. From the group $\operatorname{sl}(2, \mathbf{C})$ to gyrogroups and gyrovector spaces and hyperbolic geometry. Found. Phys., 31(11):16111639, 2001.

Jing-Ling Chen, Libin Fu, Abraham A. Ungar, and Xian-Geng Zhao. Alternative fidelity measure between two states of an N-state quantum system. Phys. Rev. A (3), 65:054303, 3, 2002.

Jing-Ling Chen, Libin Fu, Abraham A. Ungar, and Xian-Geng Zhao. Geometric observation of Bures fidelity between two states of a qubit. Phys. Rev. A (3), 65(2):024303, 3, 2002.

Jing-Ling Chen, Libin Fu, Abraham A. Ungar, and Xian-Geng Zhao. Degree of entanglement for two qubits. Phys. Rev. A (3), 65(4):044303, 4, 2002.

Jing-Ling Chen and Abraham A. Ungar. The Bloch gyrovector. Found. Phys., 32(4):531-565, 2002.

Jing-Ling Chen and Abraham A. Ungar. Introducing the Einstein metric to quantum computation and quantum information geometry. Found. Phys. Lett., 15(2):189-197, 2002.

N. A. Chernikov. The relativistic Kepler problem in the Lobachevsky space. Acta Phys. Polon. B, 24(5):927-950, 1993.

Dariusz Chruściński and Andrzej Jamiołkowski. Geometric phases in classical and quantum mechanics, volume 36 of Progress in Mathematical Physics. Birkhäuser Boston Inc., Boston, MA, 2004.

M. Chrysos. Why $\vec{p}=\gamma(v) m \vec{v}$ instead of $\vec{p}=m \vec{v}$ ? European J. Phys., 25(3):L33L35, 2004.

M. Chrysos. The non-intuitive $\frac{1}{2}$ thomas factor: a heuristic argument with classical electromagnetism. European J. Phys., 27(1):1-4, 2006.

David B. Cline. The search for dark matter. Sci. Amer., March:50-59, 2003.

Bartolomé Coll and Fernando San José Martínez. Composition of Lorentz transformations in terms of their generators. Gen. Relativity Gravitation, 34(9):1345-1356, 2002.

Christopher J. Conselice. The universe's invisible hand. Sci. Amer., February:3541, 2007.

Edmund J. Copeland, M. Sami, and Shinji Tsujikawa. Dynamics of dark energy. Internat. J. Modern Phys. D, 15(11):1753-1935, 2006.

Leo Corry. The influence of David Hilbert and Hermann Minkowski on Einstein's views over the interrelation between physics and mathematics. Endeavor, 22(3):95-97, 1998. 
Carl C. Cowen and Barbara D. MacCluer. Composition operators on spaces of analytic functions. Studies in Advanced Mathematics. CRC Press, Boca Raton, FL, 1995.

H. S. M. Coxeter. Introduction to geometry. John Wiley \& Sons Inc., New York, 1961.

H. S. M. Coxeter. Non-Euclidean geometry. Mathematical Association of America, Washington, DC, sixth edition, 1998.

C. Criado and N. Alamo. A link between the bounds on relativistic velocities and areas of hyperbolic triangles. Amer. J. Phys., 69(3):306-310, 2001.

Michael J. Crowe. A history of vector analysis. Dover Publications Inc., New York, 1994. The evolution of the idea of a vectorial system, Corrected reprint of the 1985 edition.

William C. Dickinson and Kristina Lund. The volume principle. Math. Mag., $79(4): 251-261,2006$.

P.A.M. Dirac. The evolution of the physicist's picture of nature. Sci. Amer., pages 45-53, May, 1963.

Peter Dombrowski and Jürgen Zitterbarth. On the planetary motion in the 3dim. standard spaces $\mathbf{M}_{\kappa}^{3}$ of constant curvature $\kappa \in \mathbf{R}$. Demonstratio Math., 24(3-4):375-458, 1991. Differential spaces and their applications (Pasierbiec, 1990).

Yu-Bing Dong and Ming-Fei Li. Relativistic center=ofmass vector and electric polarizability of charged pion meson. Commun. Theor. Phys. (Beijing), 41(1):63-66, 2004.

V. N. Dubrovskii, Ya. A. Smorodinskii, and E. L. Surkov. The World of Relativity. Nauka, Moscow, 1984. (Russian).

David S. Dummit and Richard M. Foote. Abstract algebra. John Wiley \& Sons Inc., Hoboken, NJ, third edition, 2004.

B. R. Ebanks and C. T. Ng. On generalized cocycle equations. Aequationes Math., 46(1-2):76-90, 1993.

A. S. Eddington. The Mathematical Theory of Relativity. Cambridge, 1924.

Jürgen Ehlers, Wolfgang Rindler, and Ivor Robinson. Quaternions, bivectors, and the Lorentz group. In Perspectives in Geometry (Essays in Honor of V. Hlavatý), pages 134-149. Indiana Univ. Press, Bloomington, Ind., 1966.

Albert Einstein. Zur Elektrodynamik Bewegter Körper [on the electrodynamics of moving bodies] (We use the English translation in [Einstein (1998)]). Ann. Physik (Leipzig), 17:891-921, 1905.

Albert Einstein. Relativity the special and general theory. New York, Crown Publishers, 1961. Trans. Robert W. Lawson.

Albert Einstein. Einstein's Miraculous Years: Five Papers that Changed the Face of Physics. Princeton, Princeton, NJ, 1998. Edited and introduced by John Stachel. Includes bibliographical references. Einstein's dissertation on the determination of molecular dimensions - Einstein on Brownian motion Einstein on the theory of relativity - Einstein's early work on the quantum hypothesis. A new English translation of Einstein's 1905 paper on pp. 123160.

Albert Einstein and Alice Calaprice. The New Quotable Einstein. Princeton 
University Press and the The Hebrew University of Jerusalem, Princeton, N.J., 2005. Enlarged Commemorative Edition Published on the 100th Anniversary of the Special Theory of Relativity; Collected and Edited by Alice Calaprice.

Artur Ekert, Marie Ericsson, Patrick Hayden, Hitoshi Inamori, Jonathan A. Jones, Daniel K. L. Oi, and Vlatko Vedral. Geometric quantum computation. J. Modern Opt., 47(14-15):2501-2513, 2000. Seminar on Fundamentals of Quantum Optics, V (Kühtai, 2000).

Francis C. W. Everitt, William M. Fairbank, and L. I. Schiff. Theoretical background and present status of the Stanford relativity-gyroscope experiment. Switzerland, 1969. in The Significance of Space Research for Fundamental Physics, Proceedings of the Colloquium of the European Space Research Organization at Interlaken, Sept. 4, 1969.

C.W.F. Everitt. New frontiers in physics. Freeman, San Francisco, 1988. in J.D. Fairbank, B.S. Deaver, Jr., C.W.F. Everitt (eds.).

William Martin Fairbank. Editorial. William Martin Fairbank (1917-1989). Nature, 342:125, 1989.

H. M. Farkas and I. Kra. Riemann surfaces. Springer-Verlag, New York, second edition, 1992.

Tomás Feder. Strong near subgroups and left gyrogroups. J. Algebra, 259(1):177190, 2003.

F. I. Fedorov. Composition of parameters of the Lorentz group. Dokl. Akad. Nauk SSSR, 143:56-59, 1962.

Rafael Ferraro and Marc Thibeault. Geometric composition of boosts: an elementary derivation of the Wigner rotation. European J. Phys., 20:143-151, 1999.

Richard P. Feynman, Robert B. Leighton, and Matthew Sands. The Feynman lectures on physics. Addison-Wesley Publishing Co., Inc., Reading, Mass.London, 1964.

J. Chris Fisher and Dieter Ruoff. A hyperbolic concurrency theorem. J. Geom., 61(1-2):46-52, 1998.

Francisco Flores. Interpretations of Einstein's equation $E=m c^{2}$. Int. Stud. Philos. Sci., 19(3):245-260, 2005.

V. Fock. The theory of space, time and gravitation. The Macmillan Co., New York, 1964. Second revised edition. Translated from the Russian by N. Kemmer. A Pergamon Press Book.

Tuval Foguel and Abraham A. Ungar. Involutory decomposition of groups into twisted subgroups and subgroups. J. Group Theory, 3(1):27-46, 2000.

Tuval Foguel and Abraham A. Ungar. Gyrogroups and the decomposition of groups into twisted subgroups and subgroups. Pac. J. Math, 197(1):1-11, 2001.

Tuval Foguel. Polar decomposition of locally finite groups. Results Math., 42(12):69-73, 2002.

Yaakov Friedman. Physical applications of homogeneous balls, volume 40 of Progress in Mathematical Physics. Birkhäuser Boston Inc., Boston, MA, 2005. With the assistance of Tzvi Scarr. 
Y. Friedman and A. A. Ungar, "Gyrosemidirect product structure of bounded symmetric domains," Results Math., vol. 26, no. 1-2, pp. 28-38, 1994.

D.H. Frisch and J.H. Smith. Measurement of the relativistic time dilation using $\mu$-mesons. Amer. J. Phys., 31(5):342-355, 1963.

R. P. Gă̌da, V. I. Tretyak, and Yu. G. Yaremko. Center-of-mass variables in relativistic Lagrangian dynamics of a particle system. Teoret. Mat. Fiz., 101(3):402-416, 1994.

John B. Garnett. Bounded analytic functions. Academic Press Inc. [Harcourt Brace Jovanovich Publishers], New York, 1981.

Maria Georgiadou. Constantin Carathéodory. Springer-Verlag, Berlin, 2004. Mathematics and politics in turbulent times, With a foreword by George Papanicolaou.

Tepper L. Gill and W. W. Zachary. Proper-time formulation of classical electrodynamics. J. Nonlinear Math. Phys., 4(3-4):418-425, 1997. Memorial volume for Wilhelm Fushchych.

Domenico Giulini. Algebraic and geometric structures of special relativity. arXiv:math-ph/0602018 v2 2006. In J. Ehlers and C. Lämmerzahl, eds. Special Relativity: Will it Survive the Next 100 Years?, to appear. SpringerVerlag, Berlin, New York.

Kazimierz Goebel and Simeon Reich. Uniform convexity, hyperbolic geometry, and nonexpansive mappings, volume 83 of Monographs and Textbooks in Pure and Applied Mathematics. Marcel Dekker Inc., New York, 1984.

Herbert Goldstein. Classical mechanics. Addison-Wesley Publishing Co., Reading, Mass., second edition, 1980. Addison-Wesley Series in Physics.

Chaim Goodman-Strauss. Compass and straightedge in the Poincaré disk. Amer. Math. Monthly, 108(1):38-49, 2001.

W. Gough. The combination of two Lorentz transformations. Nuovo Cimento Soc. Ital. Fis. B (12), 115(4):411-417, 2000.

Jeremy Gray. Ideas of space. The Clarendon Press Oxford University Press, New York, second edition, 1989. Euclidean, non-Euclidean, and relativistic.

Jeremy Gray. Möbius's geometrical Mechanics. 1993. In John Fauvel, Raymond Flood, and Robin Wilson, eds. Möbius and his band, Mathematics and astronomy in nineteenth-century Germany, The Clarendon Press Oxford University Press, New York, 1993, 78-103.

Jeremy J. Gray. János Bolyai, non-Euclidean geometry, and the nature of space, volume 1 of Burndy Library Publications. New Series. Burndy Library, Cambridge, MA, 2004. With a foreword by Benjamin Weiss, a facsimile of Bolyai's Appendix, and an 1891 English translation by George Bruce Halsted.

Marvin Jay Greenberg. Euclidean and non-Euclidean geometries. W. H. Freeman and Company, New York, third edition, 1993. Development and history.

Branko Grünbaum and G. C. Shephard. Ceva, Menelaus, and the area principle. Math. Mag., 68(4):254-268, 1995.

Guy Gugliotta. Mining for dark matter. Discover, 28(5):36-41, 2007.

Michael J. W. Hall and Malcolm R. Anderson. Invariant Hamiltonians for relativistic particles. Amer. J. Phys., 63(7):633-637, 1995. 
Paul R. Halmos. Finite-dimensional vector spaces. Springer-Verlag, New York, second edition, 1974. Undergraduate Texts in Mathematics.

Francis R. Halpern. Special relativity and quantum mechanics. Prentice-Hall Inc., Englewood Cliffs, N.J., 1968.

Robin Hartshorne. Geometry: Euclid and beyond. Undergraduate Texts in Mathematics. Springer-Verlag, New York, 2000.

Robin Hartshorne. Non-Eeuclidean III.36. Amer. Math. Monthly, 110(6):495-502, 2003.

Melvin Hausner. A vector space approach to geometry. Dover Publications Inc., Mineola, NY, 1998. Reprint of the 1965 original.

Sigurdur Helgason. Groups and geometric analysis. Academic Press Inc., Orlando, Fla., 1984. Integral geometry, invariant differential operators, and spherical functions.

David Hestenes. New foundations for classical mechanics. Kluwer Academic Publishers Group, Dordrecht, second edition, 1999.

Desmond J. Higham and Nicholas J. Higham. MATLAB guide. Society for Industrial and Applied Mathematics (SIAM), Philadelphia, PA, second edition, 2005.

V. Hlavatý. Proper time, apparent time, and formal time in the twin paradox. $J$. Math. Mech., 9:733-744, 1960.

John G. Hocking and Gail S. Young. Topology. Dover Publications Inc., New York, second edition, 1988.

I. Martin Isaacs. Algebra. Brooks/Cole Publishing Co., Pacific Grove, CA, 1994. A graduate course.

A. Nourou Issa. Gyrogroups and homogeneous loops. Rep. Math. Phys., 44(3):345-358, 1999.

A. Nourou Issa. Left distributive quasigroups and gyrogroups. J. Math. Sci. Univ. Tokyo, 8(1):1-16, 2001.

R. Jackiw. Three-cocycle in mathematics and physics. Phys. Rev. Lett., 54(3):159-162, 1985.

John David Jackson. Classical electrodynamics. John Wiley \& Sons Inc., New York, second edition, 1975.

Stanley B. Jackson and Donald Greenspan. Hyperbolic analytic geometry. Math. Mag., 28:251-269, 1955.

Daniel F. V. James, Paul G. Kwiat, William J. Munro, and Andrew G. White. Measurement of qubits. Phys. Rev. A, 64:052312-1-052312-15, 2001.

Børge Jessen, Jørgen Karpf, and Anders Thorup. Some functional equations in groups and rings. Math. Scand., 22:257-265 (1969), 1968.

Oliver Davis Johns. Analytical mechanics for relativity and quantum mechanics. Oxford University Press, New York, 2005.

Rickard M. Jonson. Gyroscope precession in special and general relativity from basic principles. Amer. J. Phys., 75(5):463-471, 2007.

Richard Jozsa. Fidelity for mixed quantum states. J. Modern Opt., 41(12):23152323, 1994.

Manuela Juárez and Mariano Santander. Turns for the Lorentz group. J. Phys. A, 15(11):3411-3424, 1982. 
Azniv K. Kasparian and Abraham A. Ungar. Lie gyrovector spaces. J. Geom. Symm. Phys., 1(1):3-53, 2004.

W.L. Kennedy. Thomas rotation: a Lorentz matrix approach. Eur. J. Phys., 23(3):235-247, 2002.

Hubert Kiechle. Theory of K-loops. Springer-Verlag, Berlin, 2002.

Michihiko Kikkawa. Geometry of homogeneous Lie loops. Hiroshima Math. J., 5(2):141-179, 1975.

Michihiko Kikkawa. Geometry of homogeneous left Lie loops and tangent Lie triple algebras. Mem. Fac. Sci. Eng. Shimane Univ. Ser. B Math. Sci., 32:57-68, 1999.

Michael K. Kinyon and Abraham A. Ungar. The gyro-structure of the complex unit disk. Math. Mag., 73(4):273-284, 2000.

Robert P. Kirshner. The extravagant universe: exploding stars, dark energy, and the accelerating cosmos. Princeton University Press, Princeton, NJ, 2000.

Adam Korányi. Around the finite-dimensional spectral theorem. Amer. Math. Monthly, 108(2):120-125, 2001.

Hans-Joachim Kowalsky. Lineare Algebra. Walter de Gruyter, Berlin-New York, 1977. Achte Auflage, de Gruyter Lehrbuch.

Werner Krammer and Helmuth K. Urbantke. K-loops, gyrogroups and symmetric spaces. Results Math., 33(3-4):310-327, 1998.

Steven G. Krantz. Complex analysis: the geometric viewpoint. Mathematical Association of America, Washington, DC, 1990.

Steven G. Krantz. A matter of gravity. Amer. Math. Monthly, 110(6):465-481, 2003.

Erwin Kreyszig. Differential geometry. Dover Publications Inc., New York, 1991. Reprint of the 1963 edition.

Eugene Kuznetsov. Gyrogroups and left gyrogroups as transversals of a special kind. Algebra Discrete Math., (3):54-81, 2003.

Claus Lämmerzahl, C.W. Francis Everitt, and Friedrich W. Hehl. Gyros, clocks, interferometers: testing relativistic gravity in space. Springer-Verlag, New York, 2001.

Claus Lämmerzahl. Relativity and technology. Ann. Phys. (8), 15(1-2):5-18, 2006.

L. D. Landau and E. M. Lifshitz. The classical theory of fields. Fourth revised English edition. Course of Theoretical Physics, Vol. 2. Translated from the Russian by Morton Hamermesh. Pergamon Press, Oxford, New York, 1975.

Jimmie Lawson and Yongdo Lim. Symmetric sets with midpoints and algebraically equivalent theories. Results Math., 46(1-2):37-56, 2004.

John M. Lee. Riemannian manifolds. Springer-Verlag, New York, 1997. An introduction to curvature.

Luis R. Lehner and Osvaldo M. Moreschi. On the definition of the center of mass for a system of relativistic particles. J. Math. Phys., 36(7):3377-3394, 1995.

Péter Lévay. The geometry of entanglement: metrics, connections and the geometric phase. J. Phys. A, 37(5):1821-1841, 2004.

Péter Lévay. Thomas rotation and the mixed state geometric phase. J. Phys. A, 37(16):4593-4605, 2004. 
Jean-Marc Levy-Leblond. Additivity, rapidity, relativity. Amer. J. Phys., 47:1045-1049, 1979.

I.V. Lindell and H. Sihova. The quotient function and its applications. Amer. J. Phys., 66(3):197-202, 1998.

James Lindesay and Tepper Gill. Canonical proper time formulation for physical systems. Found. Phys., 34(1):169-182, 2004.

Dan Loewenthal and Enders A. Robinson. Relativistic combination of any number of collinear velocities and generalization of Einstein's formula. J. Math. Anal. Appl., 246(1):320-324, 2000.

H. A. Lorentz, A. Einstein, H. Minkowski, and H. Weyl. The principle of relativity. Dover Publications Inc., New York, N. Y., undated. With notes by A. Sommerfeld, Translated by W. Perrett and G. B. Jeffery, A collection of original memoirs on the special and general theory of relativity.

Abraham S. Luchins and Edith H. Luchins. The Einstein-Wertheimer correspondence on geometric proofs and mathematical puzzles. Math. Intelligencer, 12(2):35-43, 1990.

Jair Lucinda. A general relativistic law of addition for two velocities and successive Lorentz transformations along perpendicular axes. Phys. Essays, 14(4):280-285, 2001.

P. Kevin MacKeown. Question 57: Thomas precession. Amer. J. Phys., 65(2):105, 1997.

Wilhelm Magnus. Noneuclidean tesselations and their groups. Academic Press [A subsidiary of Harcourt Brace Jovanovich, Publishers], New York-London, 1974. Pure and Applied Mathematics, Vol. 61.

Eli Maor. Trigonometric delights. Princeton University Press, Princeton, NJ, 1998.

Eli Maor. The Pythagorean theorem: A 4,000-year history. Princeton University Press, Princeton, NJ, 2007.

Jerrold E. Marsden. Elementary classical analysis. W. H. Freeman and Co., San Francisco, 1974. With the assistance of Michael Buchner, Amy Erickson, Adam Hausknecht, Dennis Heifetz, Janet Macrae and William Wilson, and with contributions by Paul Chernoff, István Fáry and Robert Gulliver.

George Marx. Is the amount of matter additive? European J. Phys., 12:271-274, 1991.

Bernard Maskit. On Poincaré's theorem for fundamental polygons. Advances in Math., 7:219-230, 1971.

John McCleary. Geometry from a differentiable viewpoint. Cambridge University Press, Cambridge, 1994.

John McCleary. Trigonometries. Amer. Math. Monthly, 109(7):623-638, 2002.

Jagdish Mehra and Helmut Rechenberg. The historical development of quantum theory. Vol. 3. Springer-Verlag, New York, 1982. The formulation of matrix mechanics and its modifications, 1925-1926.

Mordehai Milgrom. Is $95 \%$ of the universe really missing? an alternative to dark matter. Sci. Amer., August:42-50, 2002.

John Milnor. Hyperbolic geometry: the first 150 years. Bull. Amer. Math. Soc. (N.S.), 6(1):9-24, 1982. 
Charles W. Misner, Kip S. Thorne, and John Archibald Wheeler. Gravitation.

W. H. Freeman and Co., San Francisco, Calif., 1973.

Constantin I. Mocanu, Michaela I. Morega, and Alexandru M. Morega. On the Thomas rotation paradox. Hadronic J., 16(4):295-329, 1993.

Constantin I. Mocanu. On the relativistic velocity composition paradox and the Thomas rotation. Found. Phys. Lett., 5(5):443-456, 1992.

Edwin E. Moise. Elementary geometry from an advanced standpoint. AddisonWesley Publishing Co., Inc., Reading, Mass.-London-Don Mills, Ont., second edition, 1974.

C. Møller. The theory of relativity. Oxford, at the Clarendon Press, 1952.

José María Montesinos. Classical tessellations and three-manifolds. Universitext. Springer-Verlag, Berlin, 1987.

J. M. C. Montanus. Proper time physics. Hadronic J., 22(6):625-673, 1999.

David Mumford, Caroline Series, and David Wright. Indra's pearls. Cambridge University Press, New York, 2002. The vision of Felix Klein.

Péter T. Nagy. Dynamical invariants of rigid motions on the hyperbolic plane. Geom. Dedicata, 37(2):125-139, 1991.

Péter T. Nagy and Karl Strambach. Loops in group theory and Lie theory. Walter de Gruyter \& Co., Berlin, 2002.

Tristan Needham. Visual complex analysis. The Clarendon Press Oxford University Press, New York, 1997.

Alexander I. Nesterov. Three-cocycles, nonassociative gauge transformations and Dirac's monopole. Phys. Lett. A, 328(2-3):110-115, 2004.

Iain Nicolson. Dark side of the universe: dark matter, dark energy, and the fate of the cosmos. John Hopkins University Press, Baltimore, MD, 2007.

Michael A. Nielsen and Isaac L. Chuang. Quantum computation and quantum information. Cambridge University Press, Cambridge, 2000.

D. Noerdlinger, Peter. Stellar aberration and the hodograph for the Kepler problem. Amer. J. Phys., 45(12):1229-1230, 1977.

Kimonsuke Ogura. On the Lorentz transformation with some geometrical interpretations. Sci. Rep. Tôhuku Imperial Univ., 2:95-116, 1913.

Lev B. Okun. The concept of mass. Phys. Today, June:31-36, 1989.

John Oprea. Differential geometry and its applications. Prentice Hall, Upper Saddle River, NJ, USA, 1997.

J. Ryan Patrick. Euclidean and non-Euclidean geometry: an analytical approach. Cambridge University Press, 1986.

W. Pauli. Theory of relativity. Pergamon Press, New York, 1958. Translated from the German by G. Field, with supplementary notes by the author.

Dan Pedoe. Geometry. Dover Books on Advanced Mathematics. Dover Publications Inc., New York, second edition, 1988. A comprehensive course.

Roger Penrose. The geometry of the universe. In Steen, Lynn Arthur (ed.): Mathematics Today: Twelve informal essays, pages 83-125. Springer-Verlag, New York, 1978.

Roger Penrose. The road to reality: a complete guide to the laws of the universe. Alfred A. Knopf, New York, 2005.

Hala O. Pflugfelder. Quasigroups and loops: introduction, volume 7 of Sigma 
Series in Pure Mathematics. Heldermann Verlag, Berlin, 1990.

Hala Orlik Pflugfelder. Historical notes on loop theory. Comment. Math. Univ. Carolin., 41(2):359-370, 2000. Loops'99 (Prague).

Yves Pierseaux. The "fine structure" of special relativity and the Thomas precession. Ann. Fond. Louis de Broglie, 29(1-2):57-118, 2004.

H. Poincaré. Théorie des groupes fuchsiens. Acta Mathematica, 1:1-62, 1882.

J. Preskill. Clatech lecture notes. URL:

www.theory.caltech.edu/preskill/ph229, 2004.

M. H. L. Pryce. The mass-centre in the restricted theory of relativity and its connexion with the quantum theory of elementary particles. Proc. Roy. Soc. London. Ser. A., 195:62-81, 1948.

Lewis Pyenson. Relativity in late Wilhelmian Germany: the appeal to a preestablished harmony between mathematics and physics. Arch. Hist. Exact Sci., 27(2):137-155, 1982.

John G. Ratcliffe. Foundations of hyperbolic manifolds, volume 149 of Graduate Texts in Mathematics. Springer-Verlag, New York, 1994.

Wolfgang Rindler. Introduction to special relativity. The Clarendon Press Oxford University Press, New York, 1982.

Wolfgang Rindler. Putting to rest mass misconceptions. Phys. Today, 43(5):13, 1990.

Wolfgang Rindler and Ivor Robinson. A plain man's guide to bivectors, biquaternions, and the algebra and geometry of Lorentz transformations. In On Einstein's path (New York, 1996), pages 407-433. Springer, New York, 1999.

Wolfgang Rindler. Relativity: special, general, and cosmological. Oxford University Press, Oxford, New York, 2006.

M. Rivas, M. A. Valle, and J. M. Aguirregabiria. Composition law and contractions of the Poincaré group. European J. Phys., 7(1):1-5, 1986.

Alfred A. Robb. A theory of time and space. Cambridge University Press, Cambridge, 1914.

B. A. Rosenfeld. A history of non-Euclidean geometry. Springer-Verlag, New York, 1988. Evolution of the concept of a geometric space, Translated from the Russian by Abe Shenitzer.

Krzysztof Rózga. On central extensions of gyrocommutative gyrogroups. Pacific J. Math., 193(1):201-218, 2000.

Dieter Ruoff. Why Euclidean area measure fails in the noneuclidean plane. Math. Mag., 78(2):137-139, 2005.

Patrick J. Ryan. Euclidean and non-Euclidean geometry. Cambridge University Press, Cambridge, 1986. An analytical approach.

Lev V. Sabinin, Ludmila L. Sabinina, and Larissa V. Sbitneva. On the notion of gyrogroup. Aequationes Math., 56(1-2):11-17, 1998.

Marcos Salvai. On the dynamics of a rigid body in the hyperbolic space. J. Geom. Phys., 36(1-2):126-139, 2000.

R. D. Sard. Relativistic mechanics: special relativity and classical particle dynamics. W.A. Benjamin, New York, 1970.

Tzvi Scarr. Loops in relativistic dynamics. Quasigroups Related Systems, 14(1):91-109, 2006. 
B. A. Schott. On the motion of the Lorentz electron. Phil. Mag., 29:49-62, 1915.

Bertram Schwarzschild. Collision between galaxy clusters unveils striking evidence of dark matter. Phys. Today, 59(11):21-24, 2006.

Hans Schwerdtfeger. Geometry of complex numbers. Mathematical Expositions, No. 13. University of Toronto Press, Toronto, 1962.

H. Scutaru. Fidelity for displaced squeezed thermal states and the oscillator semigroup. J. Phys. A, 31(15):3659-3663, 1998.

Roman U. Sexl and Helmuth K. Urbantke. Relativity, groups, particles. Springer Physics. Springer-Verlag, Vienna, 2001. Special relativity and relativistic symmetry in field and particle physics, Revised and translated from the third German (1992) edition by Urbantke.

L. Silberstein. The Theory of Relativity. MacMillan, London, 1914.

Paul B. Slater. Comparative noninformativities of quantum priors based on monotone metrics. Phys. Lett. A, 247(1-2):1-8, 1998.

Paul B. Slater. A priori probabilities of separable quantum states. J. Phys. A, 32(28):5261-5275, 1999.

A. Sommerfeld. Über die Zusammensetzung der Geschwindigkeiten in der Relativtheorie. Physikalische Zeitschrift, 10:826-829, 1909.

Duncan M'Laren Young Sommerville. The elements of non-Euclidean geometry. G. Bell, London, 1914.

Duncan M'Laren Young Sommerville. The Elements of non-Euclidean geometry. Open Court, Chicago, 1919.

Sebastiano Sonego and Massimo Pin. Deriving relativistic momentum and energy. European J. Phys., 26(1):33-45, 2005.

Sebastiano Sonego and Massimo Pin. Deriving relativistic momentum and energy: II. three-dimensional case. European J. Phys., 26(5):851-856, 2005.

R.W. Spekkens and T. Rudolph. Optimization of coherent attacks in generalizations of the bb84 quantum bit commitment protocol. Quantum Inf. Comp., 2(1):66-96, 2002.

J.J. Stachel. History of relativity. In Twentieth century physics. Vol. I, pages 249-356. Published jointly by the Institute of Physics Publishing, Bristol, 1995. eds: Brown, Laurie M. and Pais, Abraham and Pippard, Brian.

Saul Stahl. The Poincaré half-plane. Jones and Bartlett Publishers, Boston, MA, 1993. A gateway to modern geometry.

Albert B. Stewart. The discovery of stellar aberration. Sci. Amer., March:100108, 1964.

John Stillwell. Sources of hyperbolic geometry. American Mathematical Society, Providence, RI, 1996. Pages 10 and 35.

P. Stumpff. On the relationship between classical and relativistic theory of stellar aberration. Astronom. and Astrophys., 84(1-2):257-259, 1980.

J. L. Synge. Relativity: the special theory. North-Holland Publishing Co., Amsterdam, 1965.

G. Taub. The gravity probe. Discover, 18(3):62-71, 1997.

Llewellyn H. Thomas. The motion of the spinning electron. Nature, 117:514, 1926.

V. Trimble. Existence and nature of dark matter in the universe. Ann. Rev. 
Astron. Astrophys., 25:425-472, 1987.

Ling Tsai. The relation between gravitational mass inertial mass and velocity. Amer. J. Phys., 54:340-342, 1986.

Armin Uhlmann. The "transition probability" in the state space of a *-algebra. Rep. Mathematical Phys., 9(2):273-279, 1976.

Armin Uhlmann. Parallel transport and "quantum holonomy" along density operators. Rep. Math. Phys., 24(2):229-240, 1986.

Armin Uhlmann. Parallel lifts and holonomy along density operators: computable examples using o(3)-orbits. In Symmetries in science, VI (Bregenz, 1992), pages 741-748. Plenum, New York, 1993.

Armin Uhlmann. Parallel transport and holonomy along density operators. In Proceedings of the XV International Conference on Differential Geometric Methods in Theoretical Physics (Clausthal, 1986), pages 246-254, Teaneck, NJ, 1987. World Sci. Publishing.

Abraham A. Ungar. Thomas rotation and the parametrization of the Lorentz transformation group. Found. Phys. Lett., 1(1):57-89, 1988.

Abraham A. Ungar. The Thomas rotation formalism underlying a nonassociative group structure for relativistic velocities. Appl. Math. Lett., 1(4):403-405, 1988.

Abraham A. Ungar. Axiomatic approach to the nonassociative group of relativistic velocities. Found. Phys. Lett., 2(2):199-203, 1989.

Abraham A. Ungar. The relativistic noncommutative nonassociative group of velocities and the Thomas rotation. Resultate Math., 16(1-2):168-179, 1989. The term "K-loop" is coined here.

Abraham A. Ungar. The relativistic velocity composition paradox and the Thomas rotation. Found. Phys., 19(11):1385-1396, 1989.

Abraham A. Ungar. Thomas precession and its associated grouplike structure. Amer. J. Phys., 59(9):824-834, 1991.

Abraham A. Ungar. Quasidirect product groups and the Lorentz transformation group. In Themistocles M. Rassias (ed.): Constantin Carathéodory: an international tribute, Vol. I, II, pages 1378-1392. World Sci. Publishing, Teaneck, NJ, 1991.

Abraham A. Ungar. The holomorphic automorphism group of the complex disk. Aequationes Math., 47(2-3):240-254, 1994.

Abraham A. Ungar. Midpoints in gyrogroups. Found. Phys., 26(10):1277-1328, 1996.

Abraham A. Ungar, "Thomas precession in spacetime geometries," in Robert T. Jantzen, G. Mac Keiser and Remo Ruffini (eds.): Proceedings of the Seventh Marcel Grossmann Meeting on General Relativity (Stanford Univ., July 24-30, 1994), World Scientific, New Jersey, 1996.

Abraham A. Ungar. Thomas precession: its underlying gyrogroup axioms and their use in hyperbolic geometry and relativistic physics. Found. Phys., 27(6):881-951, 1997.

Abraham A. Ungar. From Pythagoras to Einstein: the hyperbolic Pythagorean theorem. Found. Phys., 28(8):1283-1321, 1998.

Abraham A. Ungar. The hyperbolic Pythagorean theorem in the Poincaré disc 
model of hyperbolic geometry. Amer. Math. Monthly, 106(8):759-763, 1999. Abraham A. Ungar. Gyrovector spaces in the service of hyperbolic geometry. In Themistocles M. Rassias (ed.): Mathematical analysis and applications, pages 305-360. Hadronic Press, Palm Harbor, FL, 2000.

Abraham A. Ungar. Hyperbolic trigonometry in the Einstein relativistic velocity model of hyperbolic geometry. Comput. Math. Appl., 40(2-3):313-332, 2000.

Abraham A. Ungar, "The bifurcation approach to hyperbolic geometry," Found. Phys., vol. 30, no. 8, pp. 1257-1282, 2000.

Abraham A. Ungar. Hyperbolic trigonometry and its application in the Poincaré ball model of hyperbolic geometry. Comput. Math. Appl., 41(1/2):135-147, 2001.

Abraham A. Ungar. Beyond the Einstein addition law and its gyroscopic Thomas precession: The theory of gyrogroups and gyrovector spaces, volume 117 of Fundamental Theories of Physics. Kluwer Academic Publishers Group, Dordrecht, 2001.

Abraham A. Ungar. Seeing the möbius disc-transformation like never before. Comput. Math. Appl., 45:805-822, 2003.

Abraham A. Ungar. On the appeal to a pre-established harmony between pure mathematics and relativity physics. Found. Phys. Lett., 16(1):1-23, 2003.

Abraham A. Ungar. The hyperbolic triangle centroid. Comment. Math. Univ. Carolin., 45(2):355-369, 2004.

Abraham A. Ungar. The hyperbolic triangle defect. In Geometry, integrability and quantization, pages 225-236. Softex, Sofia, 2004.

Abraham A. Ungar. On the unification of hyperbolic and Euclidean geometry. Complex Var. Theory Appl., 49(3):197-213, 2004.

Abraham A. Ungar. Einstein's special relativity: Unleashing the power of its hyperbolic geometry. Comput. Math. Appl., 49:187-221, 2005.

Abraham A. Ungar. Gyrovector spaces and their differential geometry. Nonlinear Funct. Anal. Appl., 10(5):791-834, 2005.

Abraham A. Ungar. The proper-time Lorentz group demystified. J. Geom. Symm. Phys., 4:69-95, 2005.

Abraham A. Ungar. Placing the hyperbolic geometry of Bolyai and Lobachevsky centrally in special relativity theory: an idea whose time has returned. In Non-Euclidean geometries, volume 581 of Math. Appl. (N. Y.), pages 487506. Springer, New York, 2006.

Abraham A. Ungar. The relativistic hyperbolic parallelogram law. In Geometry, integrability and quantization, pages 249-264. Softex, Sofia, 2006.

Abraham A. Ungar. Newtonian and relativistic kinetic energy: analogous consequences of their conservation during elastic collisions. European J. Phys., 27(5):1205-1212, 2006.

Abraham A. Ungar. Thomas precession: a kinematic effect of the algebra of Einstein's velocity addition law. Comments on: "Deriving relativistic momentum and energy. II. Three-dimensional case" [European J. Phys. 26 (2005), no. 5, 851-856; MR2227176] by S. Sonego and M. Pin. European J. Phys., 27(3):L17-L20, 2006. 
Abraham A. Ungar. Einstein's velocity addition law and its hyperbolic geometry. Comput. Math. Appl., 53(8):1228-1250, 2007.

Abraham A. Ungar. Gyrogroups, the grouplike loops in the service of hyperbolic geometry and Einstein's special theory of relativity. Quasigroups Related Systems, 15:141-168, 2007.

Abraham A. Ungar. From Möbius to gyrogroups. Amer. Math. Monthly, 2008. in print.

Helmuth K. Urbantke. Two-level quantum systems: States, phases, and holonomy. Amer. J. Phys., 59(6):503-509, 1991.

Helmuth K. Urbantke. Lorentz transformations from reflections: some applications. Found. Phys. Lett., 16(2):111-117, 2003.

Sonia Ursini. The contributions of Gauss to hyperbolic geometry: his correspondence with the mathematicians and scientists of his era. Miscelánea Mat., (33):1-19, 2001.

C. B. van Wyk. Rotation associated with the product of two Lorentz transformations. Amer. J. Phys., 52(9):853-854, 1984.

C. B. van Wyk. Lorentz transformations in terms of initial and final vectors. $J$. Math. Phys., 27(5):1311-1314, 1986.

Vladimir Varičak. Beiträge zur nichteuklidischen geometrie [contributions to noneuclidean geometry]. Jber. dtsch. Mat. Ver., 17:70-83, 1908.

Vladimir Varičak. Anwendung der Lobatschefskjschen Geometrie in der Relativtheorie. Physikalische Zeitschrift, 11:93-96, 1910.

Vladimir Varičak. Die Reflexion des Lichtes an bewegten Spiegeln. Physikalische Zeitschrift, 11:486-587, 1910.

Vladimir Varičak. Über die nichteuklidische interpretation der relativtheorie [On the non-Euclidean interpretation of the theory of relativity]. Jahresber. Deut. Math. Verein, 21:103-122, 1912.

Vladimir Varičak. Darstellung der Relativitätstheorie im dreidimensionalen Lobatchefskijschen Raume [Presentation of the theory of relativity in the threedimensional Lobachevskian space]. Zaklada, Zagreb, 1924.

V. Vedral, M. B. Plenio, M. A. Rippin, and P. L Knight. Quantifying entanglement. Phys. Rev. Lett., 78(12):2275-2279, 1997.

Darko Veljan. The 2500-year-old Pythagorean theorem. Math. Mag., 73(4):259272, 2000.

J. Vermeer. A geometric interpretation of Ungar's addition and of gyration in the hyperbolic plane. Topology Appl., 152(3):226-242, 2005.

J. M. Vigoureux. The reflection of light by planar stratified media: the groupoid of amplitudes and a phase "Thomas precession". J. Phys. A, 26(2):385-393, 1993.

J. M. Vigoureux. Composition law of amplitudes and quantum theory. Phys. Rev. A (3), 49(3):1602-1606, 1994.

J. M. Vigoureux. Calculations of the Wigner angle. European J. Phys., 22:149$155,2001$.

Zoran Vrcelj. The noncommutativity of Lorentz transformations. I, II. Mat. Vesnik, 9(1):55-58; ibid 10(25) (1973), 33-35, 1972.

Edward C. Wallace and Steven F. West. Roads to Geometry. Prentice Hall, NJ, 
2 edition, 1998.

Scott Walter. Minkowski, mathematicians, and the mathematical theory of relativity. In The expanding worlds of general relativity (Berlin, 1995), pages 45-86. Birkhäuser Boston, Boston, MA, 1999.

Scott Walter. The non-Euclidean style of Minkowskian relativity. In The symbolic universe (J. J. Gray (ed.), Milton Keynes, England), pages 91-127. Oxford Univ. Press, New York, 1999.

Scott Walter. Breaking in the 4-vectors: the four-dimensional movement in gravitation, 1905-1910. In The genesis of general relativity, Vol. 3: Theories of gravitation in the twilight of classical physics, Part I (Jürgen Renn (ed.), (Boston studies in the philosophy of science 201). Kluwer, Boston, 2005.

Scott Walter. The scandal of spacetime. In Vesselin Petkov (ed.): Minkowski spacetime: A hundred years later, Springer, 2008.

Xiaoguang Wang. Quantum teleportation of entangled coherent states. Phys. Rev. A (3), 64(2):022302, 4, 2001.

Xiang-Bin Wang, L. C. Kwek, and C. H. Oh. Bures fidelity for diagonalizable quadratic Hamiltonians in multi-mode systems. J. Phys. A, 33(27):49254934, 2000.

Mogens Wegener. A classical alternative to special relativity. Phys. Essays, 8(3):427-433, 1995.

Eric W. Weisstein. CRC concise encyclopedia of mathematics. Chapman \& Hall/CRC, Boca Raton, FL, second edition, 2003.

E. T. Whittaker. A history of the theories of aether and electricity vol 2. $\mathrm{Hu}-$ manities Press, New York, 1973.

Harold E. Wolfe. Non-Euclidean geometry. The Dryden Press, 1945.

N. M. J. Woodhouse. Special relativity. Springer Undergraduate Mathematics Series. Springer-Verlag London Ltd., London, 2003.

Robert M. Yamaleev and Ana-Leticia Fernandez Osorio. Proper-time relativistic dynamics on hyperboloid. Found. Phys. Lett., 14(4):323-339, 2001.

Paul Yiu. The uses of homogeneous barycentric coordinates in plane Euclidean geometry. Internat. J. Math. Ed. Sci. Tech., 31(4):569-578, 2000.

Kehe Zhu. Operator theory in function spaces, volume 139 of Monographs and Textbooks in Pure and Applied Mathematics. Marcel Dekker Inc., New York, 1990.

Kehe Zhu. Spaces of holomorphic functions in the unit ball, volume 226 of Graduate Texts in Mathematics. Springer-Verlag, New York, 2005.

Jürgen Zitterbarth. Some remarks on the motion of a rigid body in a space of constant curvature without external forces. Demonstratio Math., 24(34):465-494, 1991. Differential spaces and their applications (Pasierbiec, 1990).

Karol Życzkowski, Paweł Horodecki, Anna Sanpera, and Maciej Lewenstein. Volume of the set of separable states. Phys. Rev. A (3), 58(2):883-892, 1998. 


\section{Index}

base-height identity, 298, 558

AAA to SSS conversion law, 307

AAA to SSS conversion law, Einstein, 543

addition, Einstein, 88, 401

addition, Möbius, 78, 79

addition, PV, 94

ASA-SAS conversion law, Einstein, 550

asymptotic, gyrotriangle, 312

asymptotic, vertex, 312

automorphism, 32

automorphism, extended, 111

automorphism, gyrovector space, 156

Baltzer, 2

barycenter, 503

barycentric coordinates, 126, 502

Beltrami-Klein model, 10, 221, 227, 239, 251, 408

beta identity, 94

betweenness, 168

bifurcation, 369

bifurcation principle, 237, 371

Bloch gyrovector, 375, 381, 391

Bloch vector, 12, 390, 391

Bolyai, János, 1-4, 6, 8, 12, 15, 90,

131, 227, 259, 369, 375, 399, 509

boost, 119

boost composition, Galilei, 448

boost composition, Lorentz, 448, 450 boost, Galilei, 447

boost, Lorentz, 120, 447

bright mass, 492

Bures distance, 390

Bures fidelity, 387, 388, 390, 391

Bures metric, 391

cancellation law, left-right, 55

cancellation laws, 25

cancellation laws, table, 30

cancellation, left, 32

carrier cogyroline, 197

Cauchy-Schwarz inequality, 81, 82, 89, 95, 154

center of mass, relativistic (does not exist), 501

Ceva, hyperbolic theorem, 460, 563

CMM, covariant, 471

CMS, covariant, 520

coaddition (cooperation), 18

coaddition, Einstein, 92

coaddition, Möbius, 80

coaddition, PV, 95

coangle, 237

cobetween, 211

cobetweenness, 188

cocycle, Einstein, 89

cocycle, Möbius, 80, 378

cocycle, PV, 95

cogyroangle, 362, 363

cogyroangle, alternate, 363

cogyroangle, definition, 356, 357 
cogyroangle, invariant, 359

cogyroangle, model independent, 359 cogyroautomorphic inverse property, 43

cogyroautomorphism, 23

cogyrocollinear, 188, 192, 212, 221

cogyrocurvature, definition, 244

cogyrodifference, 196, 212, 221, 358

cogyrodistance, 199, 201

cogyrogeodesic, 199, 203

cogyrogroup, 18, 45

cogyroline, 163, 164, 168

cogyroline element, 241, 243

cogyroline element, Einstein, 253

cogyroline element, Möbius, 247

cogyroline gyration transitive law,

160, 192, 199, 371

cogyroline parallelism, Möbius, 212

cogyroline transitive law, 162

cogyroline, directed, 190

cogyroline, origin-intercept, 165, 196

cogyroline, parallelism, 192

cogyrometric coefficients, definition, 243

cogyrometric, metric coefficients, 243

cogyromidpoint, 162, 199, 211, 221,

366

cogyromidpoint, definition, 198

cogyropolygonal path, 201

cogyroreflection, 365

cogyrosegment, 211

cogyrotranslation theorem, 31

cogyrotriangle, 211

cogyrotriangle equality, 200, 201, 212,

221

cogyrotriangle inequality, 158

cogyrovector, 131, 143, 356

cogyrovector parallelism, 197

cogyrovector translation, 144, 146

cogyrovector translation head, 146

cogyrovector translation,

composition, 148

cogyrovector translation, definition,

146

cogyrovector translation, head, 147

cogyrovector translation, tail, 147 cogyrovector, definition, 144

cogyrovector, rooted, 143

cogyrovector, rooted, equivalence, 144

cogyrovectors and points, 151

cohyperbolic geometry, 369

coloop property, 42

commencing, space point, 518

commencing, time, 518

commutative group, 17

compatibility condition, 426

conformal, 549, 592

congruent gyrotriangles, AAA

Theorem, 312

congruent gyrotriangles, AAS

Theorem, 296

congruent gyrotriangles, ASA

Theorem, 312

congruent gyrotriangles, SAS

Theorem, 288

congruent gyrotriangles, SsA

Theorem, 297

congruent gyrotriangles, SSS

Theorem, 288

convex hull, 127, 472

convexity, 123

cooperation (coaddition), 18, 80, 92,

95

cooperation, commutative, 52

cooperation, Einstein, 92

cooperation, Möbius, 80

cooperation, PV, 95

cosubtraction, 29, 453

covariance, 64

covariant, 64, 67, 93, 129, 416, 417,

457, 465, 469, 474

covariant, CMM, 471, 475

covariant, CMS, 520

dark energy, 531

dark mass, 492, 531

dark matter, 531

decomplexification, 78, 396

defect, gyrosquare, 337

defect, gyrotriangle, 551

defect, gyrotriangular, 300, 316

defect, identity, 301, 304 
density matrix, 375, 376, 379-381, $383,388,389$

Descartes, 3

Differentiable manifold, 259

differential geometry, 239

Doppler effect, 454

duality symmetries, 366

duality symmetry, 9, 18, 22, 23, 34,

$162,174,192,199,218,371$

dyadic symset, 7,75

ecliptic, 597

ecliptic pole, 597

Einstein, 4, 12

Einstein addition, 88, 401

Einstein coaddition, 92

Einstein gyrocentroid, 230

Einstein gyrogroup, 85

Einstein gyrometric, 222

Einstein gyrovector space, 218, 229

Einstein half, 219, 238, 442, 575

Einstein scalar multiplication, 218

Einstein three-quarter, 532

Einstein, gyrovector space, 219

Einstein, metric, 252

Einstein, metric,line element, 250

Einstein, scalar multiplication, 219

Einsteinian relativity, x, 11

Einsteinian-Pythagorean identities, 555,556

elastic collisions, classical, 496

elastic collisions, relativistic, 498

equidefect gyrotriangles, 343

equilateral gyrotriangle, 318, 319

equilateral gyrotriangle, Einstein, 544

equivalence class, 131

equivalence relation, 131

Euclid, 1-3, 6

Euclidean geometry, 5

even (a gyroautomorphism property), 40

four-vector, 122

four-velocity, 122, 454

Galilei transformation composition,
450

Galilei, transformation group, 118

gamma identity, Einstein, 88, 402

gamma identity, Möbius, 79

Gauss, Carl Friedrich, 2

Gauss, equation, 252, 254

Gaussian curvature, 243, 244, 246,

250, 254, 256, 259, 302, 303

Gibbs, Willard J., 3

group, 16

groupoid, 16

groupoid automorphism, 16

gyration exclusion theorem, 73

gyration properties, basic, 39

gyration, extended, 112

gyration, gyrotriangular, 316

gyrations, 3D-Möbius, 85

gyrations, Möbius, 81

gyrator, 5, 17, 18, 76, 104, 379

gyrator identity, 19, 59

gyro-Cartesian coordinates, 353

gyroangle between gyrorays, 273

gyroangle between gyrovectors, 271

gyroangle, definition I, 263

gyroangle, definition II, 271

gyroangle, invariant, 267

gyroangle, model independence, 264

gyroangular defect, 303, 323

gyroassociative law, left, 17, 19, 21, 42,405

gyroassociative law, mixed, 46

gyroassociative law, right, 42, 405

gyroautomorphic inverse property, 51,

$53,57,59,409$

gyroautomorphic inverse property,

restricted, 21, 27

gyroautomorphic inverse theorem, 51

gyroautomorphism, 5, 17, 32

gyroautomorphism identities, nested, 68

gyroautomorphism inversion, 39, 40

gyroautomorphism property, 154

gyrobarycentric coordinates, 126, 455, 471

gyrobarycentric coordinates,

Einsteinian, 505 
gyrobarycentric coordinates,

homogeneous, 126

gyroboost, 104, 119

gyroboost application, 106

gyroboost, composition law, 108

gyroboost, definition, 105

gyroboost, successive applications,

108

gyrocentroid, 409, 559

gyrocentroid, definition, 228

gyrocentroid, Einstein, 230

gyrocentroid, Möbius, 235

gyrocircle gyrotrigonometry, 353

gyrocircle, semi, 314

gyrocollinear, 172, 215

gyrocollinear, definition, 168

gyrocollinear, remark, 168

gyrocollinearity, 168

gyrocommutative gyrogroup, 18

gyrocommutative law, 18, 405

gyrocommutative, protection

principle, 61

gyrocosine, definition, 263

gyrocosines law, 284, 285, 287, 288,

290, 301, 329, 372, 541, 542

gyrocovariance, 64, 66-68, 175, 455, 472

gyrocovariance, definition, 175

gyrocovariant, 64, 66, 125, 129, 175,

$177,457,469,474$

gyrocovariant, CMM, 401

gyrocovariant, fidelity, 391

gyrocovariant, maps, 175

gyrocurvature, definition, 244

gyrodiameter, supporting, 196, 211

gyrodifference, 421

gyrodistance, 157

gyrodistance, gyropolygonal path, 184

gyrodistance, invariant, 540

gyrofactor, 101, 102

gyrogeodesic, 183, 409

gyrogeometric object, 423

gyrogeometry, 15, 261, 287, 356, 389,

397, 399, 400, 439, 470

gyrogroup, 17

gyrogroup cooperation, 42,43 gyrogroup extension, 102

gyrogroup PV addition, 94

gyrogroup, cooperation, 52

gyrogroup, cooperations, 22

gyrogroup, Einstein, 85

gyrogroup, group of motions, 66

gyrogroup, gyrocommutative, 17, 51

gyrogroup, Möbius, 77

gyrogroup, Möbius complex disc, 75

gyrogroup, motions, 66

gyrogroup, objects, 67

gyrogroup, PV, 93

gyrogroup, solving a system, 161

gyrogroup, two-divisible, 72

gyrogroup, two-torsion free, 72

gyroinner product, 104

gyroinvariant, 101

gyrolanguage, 5

gyroline, 163, 164, 166

gyroline element, 241, 243

gyroline element, Einstein, 250

gyroline element, Möbius, 245

gyroline element, PV, 255, 257

gyroline gyration transitive law, 172 ,

192, 213, 215, 316, 349, 371, 468

gyroline, origin-intercept, 165, 196

gyromass, 126

gyromedian, definition, 228

gyrometric coefficients, definition, 243

gyrometric, metric coefficients, 243

gyromidpoint, 172, 199, 211, 322,

366, 409

gyromidpoint $\left(p_{1}: p_{2}: \ldots: p_{n}\right), 126$

gyromidpoint identity, $75,173,332$

gyromidpoint, $\left(t_{1}: t_{2}\right), 454$

gyromidpoint, (p:q), 122

gyromidpoint, definition I, 74

gyromidpoint, definition II, 172

gyromidpoint, Einstein, 123, 230

gyromidpoint, Einsteinian mechanical

interpretation, 411

gyromidpoint, Möbius, 234

gyronorm, 104

gyroparallelepiped, 433

gyroparallelepiped addition law, 425, 440,533 
gyroparallelepiped, definition, 434

gyroparallelepiped, pre-, 430

gyroparallelepiped, pre-, definition, 431

gyroparallelepiped, relativistic law, 438

gyroparallelogram, 68, 177-179, 182,

183, 322-329, 369, 423, 444, 508

gyroparallelogram addition law, 425, 533

gyroparallelogram condition, 47, 53,

$54,178,183,325,330,343,420,422$

gyroparallelogram gyration transitive law, 183

gyroparallelogram gyrocenter, 178,

179, 332, 431

gyroparallelogram gyrodiagonals, 178,

$179,322,423$

gyroparallelogram law, 4, 180, 323,

353,402

gyroparallelogram law, Möbius, 322

gyroparallelogram symmetries, 179

gyroparallelogram, addition, 323

gyroparallelogram, addition law, 141

gyroparallelogram, defect, 324

gyroparallelogram, definition, 178

gyroparallelogram, degenerate, 178

gyroparallelogram, Einstein, 373, 418, 420-422

gyroparallelogram, gyroangles, 323, 326,327

gyroparallelogram, gyrobarycentric coordinates, 508

gyroparallelogram, gyrocenter, 420

gyroparallelogram, gyrodiagonal

gyrolength, 329

gyroparallelogram, gyrovector addition law, 65

gyroparallelogram, law, 180

gyroparallelogram, Möbius, 321, 323,

$324,328,329,373$

gyroparallelogram, opposing

gyroangles, 323

gyroparallelogram, opposite

(opposing) sides, 182 gyroparallelogram, relativistic law,

424

gyroparallelogram, sides, 326

gyroparallelogram, symmetry, 422

gyroparallelogram,addition law, 322

gyroparallelogram,Einstein, 421

gyroparallelogram,Euclidean, 421

gyroparallelogram,gyrocenter, 419

gyropolar coordinates, 353, 356

gyropolygonal gyroaddition, 23, 24,

539,595

gyroray, 266

gyroray carrier, 273

gyroray parallelism, 282

gyroray perpendicularity, 282

gyroreflection, 365

gyrosegment, 166, 211

gyrosemidirect product, 34, 35, 37-40,

$67,101,115-119,125,450,513$

gyrosines law, 294, 296, 301

gyrosines law, Einstein, 544

gyrosquare, 545

gyrosquare gyroarea, 560

gyrosquare, defect, 337

gyrosquare, definition, 336, 547

gyrosquare, pseudo, 322

gyrosum inversion, 20, 39, 43

gyrotransformation group, 114

gyrotransformation group, Einstein, 117

gyrotransformation group, PV, 117

gyrotranslation theorem, 23, 57, 63,

130, 133, 159, 167, 169, 267, 274,

$276,278,286,423$

gyrotranslation, left, 32, 168

gyrotranslation, right, 32

gyrotranslation,theorem, 133

gyrotriangle constant, 299, 371, 558, 564

gyrotriangle constant principle, 561,

$563,565,575$

gyrotriangle equality, 183, 211, 221

gyrotriangle gyroarea, 304, 558

gyrotriangle height, 298

gyrotriangle inequality, 155, 157

gyrotriangle inequality, Einstein, 89 
gyrotriangle inequality, Möbius, 81

gyrotriangle inequality, PV, 95

gyrotriangle law, 539, 577, 578,

593-595, 597, 598

gyrotriangle, congruent, 284

gyrotriangle, defect, 551

gyrotriangle, definition, 284

gyrotriangle, equilateral, 318, 544

gyrotriangle, isosceles, 311

gyrotriangle, right-gyroangled, 552

gyrotriangle, side gyrolength, 296

gyrotrigonometry, 15, 261

gyrotrigonometry, Einsteinian, 554

gyrotrigonometry, gyrocircle, 353

gyrotrigonometry, Möbius, 284

gyrotrigonometry, relativistic, 537

gyrovector, 4, 131, 132

gyrovector parallelism, 272

gyrovector perpendicularity, 272

gyrovector space, 4, 15, 153

gyrovector space isomorphism, 225

gyrovector space isomorphisms, table, 226

gyrovector space model, definition, 227

gyrovector space, Einstein, 218, 229

gyrovector space, isomorphism, 227

gyrovector space, Möbius, 233, 247

gyrovector space, motions, 156

gyrovector space, PV, 223, 236

gyrovector space, real inner product,

154

gyrovector space, relativistic, 407

gyrovector translation, 133, 135, 272,

350

gyrovector translation of gyrorays, 275

gyrovector translation, composition,

137

gyrovector translation, definition, 135

gyrovector translation, head, 136

gyrovector translation, tail, 136

gyrovector, Bloch, 375, 381

gyrovector, definition, 133

gyrovector, gyrotriangle addition law, 539 gyrovector, rooted, 132

gyrovector, rooted, equivalence, 132

gyrovector, space,Einstein, 219

gyrovectors and points, 139

Heron's formula, 303

homogeneity property, 155

hyperbolic geometry, 2-9, 12, 13

hyperbolic geometry, analytic, 5-7, 11,12

hyperbolic geometry, Beltrami-Klein model, 10

hyperbolic geometry, bifurcation approach, 369

hyperbolic geometry, Poincaré model, 8,12

hyperbolic geometry,Poincaré disc model, 214

hyperbolic Pythagorean theorem, Möbius, 290

hyperbolic Pythagorean theorem, old, 287

hyperbolic Pythagorean theorem,

Poincaré, 289

hyperbolic trigonometry, 8

hyperbolic, area, 303

inversive symmetry (a

gyroautomorphism property), 40

isomorphic, gyrogroup, 106

isomorphism, gyrovector space, 225

kinetic energy theorem, 485, 488

kinetic energy, Newtonian, 494

kinetic energy, relativistic, 495

Klein, Felix, 2, 6, 474

Lambert gyroquadrilateral, 570, 573

law of gyrocosines, 568, 569

law of gyrosine, 589

law of gyrosines, 556

law of sines, 581, 599

lever, relativistic law of, 126, 459

line, 164

line element, 251

link, Galilei, 452 
link, Lorentz, 452

Lobachevsky, Nikolai Ivanovich, 1, 3, 4, 6, 8, 12, 15, 90, 131, 227, 259, $369,375,399,509$

loop, 16, 45

loop property, 42

loop property, left, 405

loop property, right, 405

Lorentz boost, 101, 120

Lorentz gamma factor, 102, 117

Lorentz group, 101

Lorentz group, proper velocity, 508

Lorentz group, proper velocity, demystifying, 513

Lorentz transformation composition, 451

Lorentz transformation group, abstract, 117

Lorentz transformation, gyro-algebra, 445

Lorentz transformation, homogeneous, 451

Lorentz transformation, inhomogeneous, 517

Lorentz transformation, matrix representation, 447

Lorentz transformation, orthochronous, 451

Lorentz transformation, proper, 451

mass, bright, 492

mass, dark, 492

mass, intrinsic, 479, 482

mass, invariant, 471, 479, 482, 483, 492,522

mass, rest, 479, 482, 483

matter, bright, 491

matter, dark, 491

Menelaus, hyperbolic theorem, 460, 563, 565

metric, Riemannian, 247, 259

midpoint distance condition, 173, 198, 204

midpoint symmetry condition, 162 ,

173, 198, 204, 205 midpoint, Newtonian mechanical interpretation, 411

Minkowskian relativity, $\mathrm{x}, 11,454$

Mobius addition, 78, 79

Mobius coaddition, 80

Mobius complex disc gyrogroup, 75

Mobius gyrations, 81

Mobius gyrations, 3D, 85

Mobius gyrocentroid, 235

Mobius gyrogroup, 77

Mobius gyrometric, 216

Mobius gyromidpoint, 234

Mobius gyrovector space, 233, 247

Mobius matrix, 395

Mobius, scalar multiplication, 205

monodistributive law, 155

nested gyroautomorphism identity, 21

Newton, Isaac, 3

non Euclidean geometry, 369

parallel postulate, 1

parallel transport, 350, 375, 376

parallel transport, definition, 344

parallel transport, head, 347

parallel transport, successive, 349

parallel transport, theorem, 344, 348

parallel transport, Uhlmann, 391

parallelepiped, 427

parallelism, 363

parallelogram, 183, 421

parallelogram law, 3

Poincaré metric, 216

Poincaré model, 9

polar decomposition, 76, 77, 381

positive definite, 246, 251, 254

positive definiteness, 154, 208

Principia Mathematica, 3

proper time, 508, 509, 511, 514, 522

proper velocity addition, 94

proper, speed, 459

pseudo gyrodiagonal, 330, 332

pseudo gyrosquare, 322

pseudo gyrosquare, Möbius, 321

PV coaddition, 95

PV gyrogroup, 93 
PV gyrovector space, 223, 236

PV model, 11

PV scalar multiplication, 223

Pythagorean theorem, Einstein, 554

Pythagorean theorem, hyperbolic, 554

quantum computation, 375

qubit, 375

rapidities, 90, 554

rapidity, 90

real inner product space, 97

reflection, 365

relativistic gyrotrigonometry, 537

relativistic invariant mass, 477, 479

relativistic light cone, 516

relativistic mass identities, 461

Riemannian line element, 243

Riemannian line element,

Beltrami-Klein, 252

Riemannian line element, Euclidean metric, 240

Riemannian metric, 247

Riemannian, dual line element,PV

gyrovector space, 258

Riemannian, line element, 259

Riemannian, line element,Einstein

gyrovector space, 252, 254

Riemannian, line element,PV

gyrovector space, 256

Riemannian, metric, 259

Riemannian, surface, 254

rooted gyrovector, 345

Saccheri gyroquadrilateral, 566, 569 scalar associative law, 154, 408 scalar distributive law, 154, 408 scalar field, 457 scalar multiplication, Einstein, 218 scalar multiplication, Möbius, 205 scalar multiplication, PV, 223 scaling property, 154, 267, 361

semidirect product, 450

Sommerville, Duncan M.Y., 1, 2, 4

SSS to AAA conversion law, 542

SSS to AAA conversion law, Einstein, 542

SSS to AAA conversion law, Möbius, 296

stellar aberration, 577

stellar aberration formulas, 596

stellar parallax, 577, 597

surjective, 52, 207, 208

symmetric product, 382

symmetric sum, 382

system of two equations, 160

system, disintegrated, 522

table, cancellation laws, 30

table, gyrocommutative gyrogroup

identities, 72

table, gyrogroup identities, 51

table, gyrovector space isomorphisms, 226

table, Riemannian line elements, 259

tessellation, 367

Thales of Miletus, 1

Thomas gyration, 5, 17, 403

Thomas half, 406

Thomas precession, 5, 403

Thomas precession, notorious, 407

transformation group, Galilei, 118

triangle area, 304

triangle area principle, 563, 575

two-sum identity, 156, 174-176, 199, 383,385

vector, 3

vector space, real inner product, 153 